\title{
Learning to read in English: Comparing monolingual English and bilingual Zulu- English Grade 3 learners
}

\begin{abstract}
The acquisition of reading skills of 100 monolingual English and 100 bilingual ZuluEnglish third-grade learners was investigated by measuring their phonological- and reading skills. Little research exists on how the presence of a spoken-only Zulu (L1) could influence the English (L2) reading acquisition process. PA tasks were correlated with reading measures in English as an $L 1$ and $L 2$, but significant differences were found on all of the measures; implying overall support for use of PA measures for reading achievement in L1 English monolinguals and in the English (L2) of bilingual children, but with the understanding that L1 Zulu spoken proficiency and L2 English-only instruction influences the underlying repertoire of PA skills used for L2 English reading acquisition, different from that of the L1 English reading acquisition process. The implications of these findings for $\mathrm{L} 2$ reading development and using phonological measures across cultural-linguistic groups, for educational purposes, are discussed.
\end{abstract}

Keywords: bilingualism; phonological awareness; orthographic transparency hypothesis; word reading; reading comprehension.

Diana Soares De Sousa \& Yvonne Broom, Department of Psychology, University of Witwatersrand, E-mail: dianasoaresdesousa@yahoo.co.uk and Yvonne.Broom@wits.ac.za

South African Journal of Childhood Education | 2011 1(1): 1-18 | ISSN: $2223-7674$ | ○ UJ
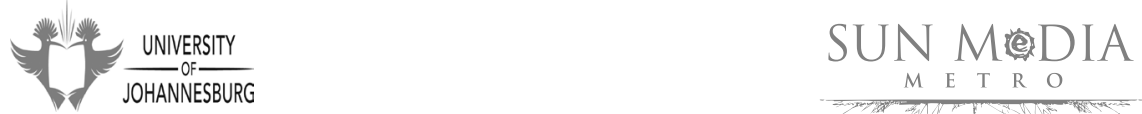


\section{Introduction: academic achievement, phonological skills, and bilingualism}

In South Africa the complex issue of language in education is historically and politically motivated and gives rise to a variety of learning experiences (Heugh, 2010). South Africa has 11 constitutionally recognised languages, yet learning to read in a language that is not their home language is an increasingly common experience for many bilingual and multilingual children. Bilingualism is defined as the ability to speak two languages, but the reality of this is more complex as the two languages may not be used with the same degree of proficiency, one may dominate over the other, and the language of reading may not be the first-language acquired (Baker, 2006). In South Africa parents are permitted to choose the language in which their child is educated (Department of Education, 2002), but the majority of parents demand that their children are educated in English (Heugh, 2010). This is partly due to global prestige of English as a medium of international communication, language of business, and prerequisite for employment (Buthelezi, 2003). In particular, the post-apartheid influx of Zulu-speaking individuals from rural to large urban areas where English dominates has led to many children in the Gauteng region beginning their education in English. It is generally accepted that this preference is educationally detrimental since the excolonial language, such as English, French or Portuguese is not known well-enough to allow for the full development of knowledge and cognitive skills (cf. Benson, 2002 for a review). However, many parents are not cognisant of the relationship between the child's first language and the language of literacy instruction.

According to Cummins' $(1979,1999)$ interdependence model, literacy instruction in the first-language facilitates appropriate development of cognitive academic skills and this forms the foundation for conceptual skills necessary for successful development of those skills in a second-language. Bilingual Zulu-English speaking children often have early verbal input in Zulu and English is introduced once they enter school and develops subsequently through English literacy instruction (Verhoeven, 2007). The language situation of these children is termed both emergent bilingual and English second language learners (EL2) as they first encounter a new language when they go to school and have limited oral proficiency in that language (Bialystok, Luk \& Kwan, 2005), as opposed to other bilingual learners who have spoken both languages before and after scholastic instruction begins. Internationally, researchers agree that academic success depends on a learner's language learning ability (Bialystok, 2007). In the event that the learner's language skills are not well-developed, he/she will experience the scholastic demands of the educational curriculum as a learning barrier and will struggle to meet these demands.

The development of oral language skills serves as a foundation for reading development and its composite skills. For the acquisition of reading skills, the development of Phonological Awareness (PA) has been shown to be of fundamental importance. Studies have shown that PA is related to reading performance in monolingual English speaking children (Bradley \& Bryant, 1983; Goswami \& Bryant, 
1990; Snow, Burn \& Griffin, 1998; Chiappe \& Siegel, 1999). Recent studies have shown that PA is also related to second-language English reading performance (Chiappe \& Siegel, 1999; Hulme \& Snowling, 2009; Low \& Siegel, 2005). PA refers to a child's various independent levels of awareness that spoken words can be broken down into smaller units of sounds, such as onsets, rimes and phonemes (Treiman \& Zukowski, 1996). Phonemic awareness refers to the ability to reflect on and manipulate phonemes. It develops and is enhanced by children's exposure to literacy instruction, and is linked to literacy achievement, because it reflects sound-analysis skills that underlie reading and spelling (Ehri et al., 2001; Hulme \& Snowling, 2009).

The development of PA involves a developmental progression from the larger units of syllables, the onset-rime level, to the smaller phoneme level (Goswami, 2000). Reading and writing require the child to learn the abstract relationship between spoken sounds and written symbols, and children who find it difficult to make phonemic judgments may struggle to learn to read. PA has been measured using a variety of tasks varying in target content, task demand and degree of explicit vs. implicit awareness required. Target content ranges from syllable awareness (least difficult), through onset-rime awareness, and phoneme awareness (most difficult). The processes involved include both synthesis (e.g. blending sounds into a word) and analysis (e.g. breaking down a provided word). Analysis tasks are further subdivided into identification (e.g. indicating the sound located in a particular word in a given position), segmentation (e.g. separating phonemes in a given word or counting phonemes in a given word), and deletion (pronouncing a word after removing a given sound). PA tasks also differ in degree of explicit awareness vs. implicit awareness required; implicit awareness is necessary for indicating which of the three words has a different initial (onset, e.g. /b/ in the word /bat/) or final sound (rime, e.g. /at/ in the word bat), more explicit awareness is needed to identify or reverse the order of phonemes, e.g. /b//la/-/t/. All PA tasks have been found to consistently correlate with reading and spelling achievement; with the simpler PA being appropriate and showing more variability in younger children, whereas the more complex ones are more appropriate for older children (cf. Anthony \& Lonigan, 2004).

Bialystok's (2002) theoretical framework identifies oral language proficiency (OLP), PA and knowledge of Grapheme-Phoneme Correspondence (GPC) rules as phonological processing skills related to reading performance in monolingual English speakers, as well as in bilingual and second language learners. However, EL2 children have to acquire these abilities in both their first and second languages. EL2 children may have different levels of oral language proficiency (OLP) in their two languages; often their OLP is higher in L1 than in L2. In addition, this model argues that orthographic knowledge and reading skills acquired in the EL2 child's L1 transfers to their L2 when an EL2 child learns to read in his/her L2. It is argued that the relationship between the writing systems of the two languages spoken by a bilingual individual determines the commonality of cognitive skills that are required for reading. Consistent with this, Bialystok, Luk and Kwan (2005) assessed Spanish-English bilingual children whose two languages shared an alphabetic writing system. They found that the word reading 
of EL2 learners was facilitated by their first language reading skills. PA skills were transferred across languages and word recognition scores were positively correlated across languages. They concluded that the differences in reading progress between the two languages, as well as the extent to which reading skills transferred from the one language to the other, depended on the similarity of GPC rules across the two languages. That is, Spanish and English share many similarities (e.g. the sounds represented by the letters $b, c, d, f, I, m, n, p, q, s$ and $t$ ), but vowels look the same in Spanish and English, but represent different sounds. Therefore, unfamiliar phonemes, such as seven English vowel sounds and their various spellings are challenging for Spanish-speaking children learning to read in their English (L2).

Differences between the orthography of Zulu and English could affect the organisation of phonological representations (Cope 1983; Mesthrie, 1995; Naidoo, 2003; Poulos \& Msimang, 1998). The Zulu language is orthographically transparent, with consistent mappings between letters and sounds (Poulos \& Msimang, 1998). There are four syllable types: V, VC, CV and CVC (Suzman, 1996) with regular and consistent GPC rules. In contrast, English has an opaque orthography with highly inconsistent and irregular GPC mappings so individual graphemes may represent a number of different phonemes in different words (Suzman, 1996). In addition, some research suggests that different intra-syllabic phonological structures are important for Zulu and English. For instance, Greenop (2004) reported that English readers were better at pseudo-word reading when the rime segment occurs relatively frequently in written English. This effect was much weaker in children in semi-rural areas learning to read Zulu, which suggests that rime may not be so salient for these children. Given this, the effect of rime on reading achievement in the L2 English of emergent bilingual, Zulu-English speaking children is an important issue to consider. If onset and phoneme levels of PA are more salient than rime, then once these are acquired in the emergent bilinguals' L1 these might have a transfer effect on L2 English reading achievement.

Very little research exists on the reading acquisition of bilingual children who attend literacy instruction in their L2 and not in their first-language. Chiappe and Siegel (1999) compared the PA and reading acquisition skills of grade 1 bilingual Punjabi (L1) children who were learning to read in their English (L2) with native-English speaking on measures of word recognition and phonological processing. These authors found no significant differences between the two groups and concluded that the English (L2) children had skills in phonological processing and reading skills comparable to those of their L1-English speaking peers. It is not simply that PA and reading skills in English need to be considered, but also if the presence of a spoken L1 makes the processes of the L2 reading acquisition process, different to the L1 reading acquisition process (Bernhardt, 2003). This potentially radical interpretation means that one has to assume that the reading acquisition process varies across English as an L1 and English as an L2 context.

Past research has provided evidence that the transparency of a language's orthography influences the strategies that children use when learning to read in that language, as well as the relative ease with which reading skills are acquired (Cardoso- 
Martins, 1995; Spencer \& Hanley, 2003; Greenop, 2004; Hulme \& Snowling, 2009). However, there has been very little research into the PA abilities important for reading achievement in children whose native language is Zulu (a transparent orthography), but who are receiving English literacy instruction (an opaque orthography).

This situation is common in South Africa. The following question then arises:

Do children in this situation develop adequate PA skills important to reading achievement in their second-language with their first prolonged exposure to English occurring only at school?

\section{Research hypotheses}

It is held that differences in the Zulu and English orthographies produces differences in PA abilities and reading strategies (Greenop, 2004). Thus, it is expected that emergent bilingual, Zulu-English speaking children who have spoken, but no written language proficiency in Zulu (L1), yet are required to learn to read English via English-only reading instruction, will perform differently on PA and reading tasks administered in English when compared with their first language English speaking peers (Bernhardt, 2003). This has important implications for reading instruction, as well as for the remediation of any reading difficulties or reading developmental disorders. In addition, this study sought to contribute to research on the role of bilingualism on children's reading development, extending existing cross-linguistic studies (Bialystok et al., 2005).

\section{Method}

\section{Research design}

This study utilised a non-experimental, ex-post facto, comparison group design. Cognitive processing in pre-existing groups, without manipulation of independent variables or randomisation of participants into samples was undertaken.

\section{Participants}

One hundred (100) monolingual, English speakers and 100 emergent bilingual, ZuluEnglish Grade 3 learners were selected from four public primary schools within the same demographic urban area in Gauteng, South Africa. All reading instruction materials at the schools were in English. Schools were matched for classroom size and teaching methods (combination of whole words and phonics). These schools are administered by the same educational authority, and follow the same curriculum. According to the South African National Education Policy for Grade 1 through to Grade 3, children are taught the letters of the alphabet, short stories, and poems with a strong emphasis on a communicative language approach (Department of Education, 2002). Public primary schools in South Africa only accept children that live in their immediate surrounding areas. Thus, these children may be considered equivalent from a socio-economic viewpoint. 
All of the teachers in the 10 participating schools spoke English as their first language, had similar teaching backgrounds, and had similar teaching experiences. To determine the equivalence of the classroom experiences of participants from different schools an experimental check of teacher-classroom quality was conducted using the Early Language and Literacy Classroom Observation (ELLCO; Smith et al., 2002). The ELLCO, measures the quality of language and literacy support in the classroom environment including the organisation of the classroom, opportunities for children's reading initiative, classroom management, the presence and use of books, and embedded writing opportunities. These results confirmed that classrooms were comparable across a range of quality indices $(F(1,99)=.56, p>.05)$. EL2 participants were selected as being predominantly Zulu-speaking at home, with their first prolonged exposure to English occurring only at school. Responses to parental home language exposure and questionnaires indicated that none of these learners had been exposed to print materials in Zulu nor had they received any reading instruction in Zulu. Responses to parental home language exposure and use questionnaires indicated that the monolingual English-speaking children were not exposed to any other language at home.

The EL1 group (Mage $=9.40, \mathrm{SD}=.72$ ) comprised 46 boys and 53 girls, while the EL2 group (Mage $=9.34, S D=.60$ ) consisted of 58 boys and 42 girls. There were no significant differences in age $(F(1,99)=.56, p>.05)$ or gender $(F(1,99)=5.95, p>.05)$ nor in general cognitive ability, as assessed on the Raven's Coloured Progressive Matrixes (RCPM, Raven, Raven \& Court, 1998) $(F(1,99)=2.23, p>.05)$.

\section{Materials}

- Onset-Rime Detection-Level of PA: Bradley and Bryant's (1983) English sound categorisation test was used to assess onset and rime awareness-levels of PA. Each learner was presented with three words. For example, in the onset awareness test, the examiner says, "rot, rod, box" and the learner is required to say, "box". Similarly, for the rime awareness test, the examiner says, "mop, hop, tap" and the learner is required to say, "tap". Ten onset and 10 rime items were administered. Reliability was calculated using Cronbach's alpha and results of $\alpha=.85$ and $\alpha=.87$, for the English, onset and rime tests, respectively, for the monolingual Englishspeaking children were found. Cronbach's alpha was calculated as $\alpha=.88$ and $\alpha=$. 80, for the English, onset and rime tests, respectively, for the emergent bilingual, Zulu-English speaking children.

- Phoneme Deletion-Level of PA: Rosner's (1979) test of auditory analysis skills, which taps the ability to delete English, syllables, phonemes and phoneme-in-a-blend was administered. For instance, the examiner says to the learner "say sunshine without the "shine"" and the learner is required to respond "sun". Fifteen items were administered. Cronbach's alpha for the EL1 group was calculated as $\alpha=.95$ while for the EL2 group it was $a=.91$. 
- Word reading: English word reading was assessed using the Weschler Individual Assessment Test-II (WIAT-II, Weschler, 2005), which taps items that are typically found in early grade reading texts (Grade 1-3), e.g. 'the', 'ocean' and 'size'. This information was confirmed by the Grade 3 teachers. Eighty-five reading items were administered and the number of words read correctly was the measure of word reading ability. Cronbach's alpha for the EL1 group was calculated as $\alpha=.87$ and $\alpha=.79$ for the EL2 group.

- Reading comprehension tests: English reading comprehension was assessed using two-age appropriate unrelated passages of the English, Neale Analysis of Reading-Revised-II (NARA-Revised-II, Neale, 1997). The Neale measures ability to comprehend text in a meaningful context: sentences vary in length, complexity and vocabulary level. A total of 16 questions were asked, eight for each passage and the number of correct answers was the measure of comprehension ability. Cronbach's alpha was calculated as $\alpha=.88$ for the EL1 and $\alpha=.80$ for the EL2 group.

\section{Procedure}

Participants were tested during the middle of their Grade 3 school year in a quiet room at their respective schools. All tests were administered by a fluent English speaker. Testing took place over two sessions to avoid fatigue. Appropriate informed consent from parents and school authorities was obtained for all of the participants. In addition, learners gave their written consent to participate in the study. Ethical clearance was obtained from the relevant University Ethics Committee (Protocol Number Ho 91010).

\section{Results}

A Kolmogorov-Smirnov goodness of fit test found the data to have a normal distribution. Similarly, Levene's test for the homogeneity of variance indicated that the variances within the groups were equal (Coolican, 2004). Therefore, parametric statistics were used to analyse the data. The means and standard deviations for all of the measures are presented in Table 1.

Table 1: Performance of EL1 the EL2 learners on the phonological awareness and reading tasks

\begin{tabular}{|l|c|c|c|c|c|}
\hline \multicolumn{2}{|c|}{} & \multicolumn{2}{c|}{ EL1 (N=100) } & \multicolumn{2}{c|}{ EL2 (N=100) } \\
\hline Onset detection & 10 & $M$ & SD & $M$ & SD \\
\hline Rime detection & 10 & 7.94 & 1.72 & 6.44 & 2.24 \\
\hline Phoneme deletion & 15 & 7.50 & 2.11 & 4.80 & 2.17 \\
\hline Word reading & 85 & 55.36 & 10.45 & 42.39 & 11.18 \\
\hline Comprehension & 16 & 10.14 & 4.89 & 5.83 & 4.05 \\
\hline
\end{tabular}




\section{PA, reading and reading comprehension accuracy}

A multivariate analysis of variance (MANOVA) between phonological processing and word reading and reading comprehension measures demonstrated that performance across these constructs was significantly different for the EL1 and EL2 groups (Wilks' Lambda $(F(5,191)=6.55, p<.001)$. Given this result, (e.g. cf. Coolican, 2004), five analyses of variances were calculated (i.e. between EL1 PA and EL2 PA; EL1 word reading and EL2 word reading, and EL1 reading comprehension and EL2 reading comprehension). Type I error rates among the five comparisons were controlled through Bonferroni adjustments $(5 \times 2=10 ; .05$ divided by $10=.005)$. Inspection of the ANOVA scores shown in Table 2 demonstrated that all of the tests were statistically significant at $(p<.001)$. Tests of statistical significance, such as MANOVA are, in part, influenced by sample sizes. To provide an increased understanding of the size of significant differences among groups, effect size is reported in this study. Effect sizes use standard deviations, rather than standard errors, and are thus not influenced by the sample size. Differences in effect size can be considered a standardised measure of group difference (Cohen, 1988). All of the statistically significant results in Table 2 demonstrated a moderate-to-large effect size $(d=.50$ through to $d=.80$ and above) respectively, following Cohen's (1988) guidelines. For both groups of learners, reading comprehension demonstrated a higher effect size (1.20) than word reading (.96), with the degree of difference being greater for the EL2 than for the EL1 group. In addition, the effect size for PA, particularly rime (1.20), demonstrated a large effect size, with the degree of difference greater for the EL2 than for the EL1 group.

Table 2: MANOVA results comparing EL1 and EL2 learners' performance on the phonological awareness and reading tasks

\begin{tabular}{|l|c|c|c|c|}
\hline \multicolumn{1}{|c|}{ Dependent variable } & $N$ & d.f. & F value & Effect size $d$ \\
\hline Onset detection & 100 & $(1,98)$ & $21.13^{* * *}$ & .75 \\
\hline Rime detection & 100 & $(1,98)$ & $35.55^{* * *}$ & 1.26 \\
\hline & & & & .53 \\
Phoneme deletion & 100 & $(1,98)$ & $9.65^{* * *}$ & .96 \\
\hline Word reading & 100 & $(1,98)$ & $27.86^{* * *}$ & 1.20 \\
\hline Comprehension & 100 & $(1,98)$ & $28.92^{* * *}$ & \\
\hline
\end{tabular}

$* * * p<.001$.

\section{The relationship between PA and literacy measures in English as an L1 and L2}

Correlation analyses were then conducted and the results are shown in Table 2. Inspection of these scores showed that for the EL2 group there were several significant, positive moderate associations between PA and reading measures. There were also significant, positive moderate associations between EL1 PA and EL1 reading measures. However, there is an additional factor that must be considered when interpreting the strength of a relationship between two variables, namely the significance of 
the correlation. By applying Fisher's z transformations (cf. Hays, 1994), all pair-wise comparisons between the correlations can be tested. Significant correlations were found for onset-rime ( $r=.60$ vs. $r=.37 ; Z r=2.595, p<.001)$ and phoneme-reading comprehension ( $r=.35$ vs. $r=.56 ; Z r=3.567, p<.001)$.

Table 3: Correlation of phonological awareness and reading tasks for the EL1 (shaded area) and EL2 learners

\begin{tabular}{|l|c|c|c|c|c|}
\hline \multicolumn{1}{|c|}{ Variable } & Onset & Rime & Phoneme & Word reading & Comprehension \\
\hline Onset & & $.37^{* * *}$ & $.37^{* * *}$ & $.36^{* * *}$ & $.31^{* * *}$ \\
\hline Rime & $.60 * * *$ & & $.32^{* * *}$ & $.58^{* * *}$ & $.51^{* * *}$ \\
\hline $\begin{array}{l}\text { Phoneme } \\
\text { deletion }\end{array}$ & $.24^{* *}$ & $.30^{* *}$ & & $.64^{* * *}$ & $.56^{* * *}$ \\
\hline Word reading & $.21^{*}$ & $.38^{* * *}$ & $.27^{* *}$ & & $.67^{* * *}$ \\
\hline Comprehension & $.31^{* * *}$ & $.31^{* * *}$ & $.35^{* * *}$ & $.66^{* * *}$ & \\
\hline
\end{tabular}

$* * p<.01$

$* * * p<.001$.

To investigate the specific nature of concurrent predictors of reading tasks, PA predictors' were entered into a stepwise multiple regression analysis, separately for word reading and reading comprehension and separately for the English (L1) and English (L2) groups. In stepwise multiple regression analyses, complex inter correlations allow predictor variables that do not contribute significantly (or only weakly) to the criterion variable to be removed as stronger predictors are entered. Criteria for variable entry and removal are as follows: probability of $F$ to enter .05 and probability of $F$ to remove .10 (Coolican, 2004). For English word reading, in the EL1 group, rime detection level of PA was the only significant predictor, explaining $19 \%$ of the variance, $(F(3,96)=7.26, p<.001)$. For English word reading, in the EL2 group, two variables explained $56 \%$ of the variance. Phoneme deletion level of PA accounted for $42 \%$ of variance, and rime detection level of PA added a further $14 \%$ to the prediction, $(F(3,96)=41.24, p<.001)$. For English reading comprehension, in the EL1 group, phoneme deletion level of PA was the only significant predictor, explaining $3 \%$ of the variance, $(F(3,96)=7.70, p<.001)$. For English reading comprehension, in the EL2 group, two variables explained $44 \%$ of the variance. Phoneme deletion level of PA accounted for $30 \%$ of variance, and rime detection level of PA added a further $14 \%$ to the prediction, $(F(3,96)=25.24, p<.001)$.

\section{Discussion}

The aim of this study was to explore whether emergent Zulu-English bilinguals with spoken Zulu (L1) proficiency only and English (L2) reading instruction, perform 
differently on PA, word reading and reading comprehension tasks in their English (L2), relative to their monolingual English (L1) speaking peers. The results indicated that, for both the EL1 and the EL2 learners, phonological processing skills were moderately, positively associated with both word reading and reading comprehension tasks. Thus, phonological processing skills are related to reading outcomes in English as an L1 and L2. This result is consistent with studies that have reported that similar cognitive processes underlie first-language and L2 English reading attainment, which in turn has led to a tendency to explain English (L2) reading acquisition based on theories of English (L1) development (e.g. Chiappe \& Siegel, 1999; Low \& Siegel, 2005).

However, this conclusion is clearly premature. That is, the present study also found significantly different correlations for onset-rime and phoneme deletion-reading comprehension, as well as found statistically significant differences between PA, word reading and reading comprehension measures in EL1 and EL2 group. This pattern of findings, suggests that different phonological processing skills predict reading tasks in EL1 and EL2. This then implies that reading acquisition in an EL1 and reading development in an EL2 context involves different strategies based on different underlying phonological processing skills; with L1 and L2 language background and orthographic transparency, and level of bilingualism exerting an influence on the reading acquisition process of EL2 children.

Zulu uses the same Latin-based alphabetic script as English, but it has a high degree of orthographic transparency and highly salient syllables: it is multisyllabic and has clear syllabic boundaries (Suzman, 1996). The EL2 data suggests that transfer occurs and that it might be influenced by a bilingual child's L1. Notably, the correlation between onset level of PA and English reading measures in the EL2 group is similar in size to the correlation between the onset level of PA and English reading measures in the EL1 group. In addition, the PA of the EL2 group seems to be consistent with PA for Zulu monolingual children, finding onset and phoneme levels of PA more accessible than rime (Greenop, 2004).

Further, if onset and phoneme levels of PA are more salient than rime, then once these are acquired in the EL2 groups' L1 these might have a transfer effect on their L2 English reading achievement. Thus, the effect of bilingualism can be found on tasks that contrast the most across the dissimilar languages of EL2 children. Furthermore, some research suggests different intra-syllabic phonological structures are important for Zulu and English reading achievement. Greenop (2004) reported that English readers were better at pseudo-word reading when the rime segment occurs relatively frequently in written English. This effect was much weaker in children in semi-rural areas learning to read Zulu, which suggests that rime may not be so salient for these children. Similarly, Cardoso-Martins (1995) reported that the sensitivity to global phonological similarity did not seem to make a distinctive contribution to literacy acquisition in Portuguese, which also has a regular, transparent orthography than English. Rime holds a special status in English, because there is a higher percentage of rime neighbours in English (i.e. for the target word 'cat', there are more words that 
have the same sounds in the rime, e.g. 'bat', 'chat', 'flat' and 'sat' than at the level of individual phonemes, e.g. 'too', ‘two' and ‘to' (Goswami, 2000).

In contrast, the close correspondence between letter-sound mappings in spoken Zulu means that rime is not salient (Suzman, 1996). This implies that despite access to L2 English literacy instruction, EL2 children use L1 Zulu spoken only phonological structures in their English (L2) reading acquisition process. Further, since the skills required for reading in Zulu and English differ, particularly at the level of rime, relying on a letter-by-letter decoding strategy is not an effective strategy as English is only a partially phonetic language and most words cannot be decoded using sound alone. Hence, the emergent Zulu-English children are meeting with less English (L2) reading success than if they had to read in a transparent orthography, such as Zulu.

Wimmer (1993) has argued that learning to read in a transparent orthography may be characterised by more rapid word-decoding skills than when learning to read an opaque orthography. Consistent with this claim, Wimmer and Goswami (1994) found that Austrian children who were learning to read in German rapidly surpassed their English peers at a relatively early age in the reading acquisition process. Thorstad (1991) observed a very similar pattern of findings in a study that compared reading achievement in Italian children aged 6-11 years with those made by their English peers, as did Spencer and Hanley (2003) in their study comparing reading achievement in Welsh children aged 5-8 years to that of same aged English children. The latter study, matched their Welsh-medium and English-medium participants by catchment area, by classroom size, and by reading curriculum and instruction time; hence, it appears that the significance and magnitude of between-language differences are not an artefact of methodology.

In addition to differences in the reading progress between two dissimilar languages, bilingual children have been argued to be able to transfer skills from one language to another, suggesting that bilingualism may have facilitating effects on children's literacy acquisition. In a study by D'Anguilli, Siegel and Serra (2001) on the development of reading in Italian-English bilingual children, exposure to literacy in a language with predictable GPC's, such as L1 Italian, in conjunction with L2 English, bilingualism was associated with enhanced phonological skills and reading ability in L2 English. In this study, however, the PA and reading performance of the EL2 group was significantly poorer than that of their EL1 group. Thus, the emergent bilinguals' Englishonly educational environment may be incapable of bridging the gap between EL1 and EL2 PA and reading skills. But, the implication is that if reading instruction is provided in Zulu allowing for PA development in Zulu, together with familiarity of written Zulu, and explicit reading instruction of rime level of PA in English, then this would indirectly accelerate their PA importance for reading achievement not only in their Zulu (L1), but also their English (L2), via transfer of PA from Zulu to English reading and lead to the development of non-phonological reading strategies that are required for reading in L2 English. 
Given similar demographics, language characteristics, and context where one language is given oral/verbal-only status and the other educational status, findings from early bilingual development can be generalised from one situation to another (cf. also Cummins, 1999 on this point). However, the use of cross-sectional and correlational data, between the EL1 PA and reading measures, as well as between the EL2 PA and reading measures, means that differences in these relationships cannot be interpreted as causal, or that L1 Zulu spoken proficiency has a directional effect, but rather interpreted as the degree to which a measured change in one construct co-varies with that measured change in another construct. Replication of this study in a longitudinal study is warranted so that the significance of bilingualism for PA skills used for academic achievement can be fully established. Further, this study did not measure PA or reading in Zulu (L1), but only English (L2), and thus inclusion of a monolingual Zulu group is recommended. Nonetheless, naturalistic studies, such as this study, present a first step towards a better understanding of emergent bilingualism and children's L2 English PA and literacy achievement. Additionally, the concepts of language interdependence, emergent bilingualism, and PA have important implications for teaching and facilitating of learning, and may eventually be of benefit to bilingual learners being educated in their EL2.

\section{Implications: pedagogical, curriculum and evaluation in education}

The findings of this study broadly support the claim that PA skills are important for reading success in an EL1 and reading success in an EL2 (Chiappe \& Siegel, 1999; Low \& Siegel, 2005). This study extends this to children whose L1 is Zulu, but are nonetheless being educated in English. PA skills are important to teach for reading achievement. However, PA is not a unitary concept - different PA skills may influence reading achievement. In this study, rime level of PA was well-achieved, and predictive of reading tasks in EL1 group than in the EL2 group. Therefore, for pedagogy, this level of PA needs to be explicitly taught to EL2 children to improve their reading ability, especially if rime is not salient in these children's L1. In addition, PA is related to exposure to print materials in teaching in a L1 and L2 (Ehri et al., 2001). Thus, exposing EL2 children with a transparent $L 1$ to nursery rhymes in English may help facilitate sensitivity to rime level of PA important for learning to read in EL2.

For curriculum, the gap between English word reading and English reading comprehension for both groups of learners needs to be addressed. The Outcomesbased Education System reading curriculum in South Africa emphasises self managed and directed learning to access a range of information sources (Department of Education, 2002). If this were the case then word reading and reading comprehension should be achieved at similar levels. Yet, both groups of learners have difficulty learning by reading, and this could have a possible hampering effect in access to various subject areas of the South African curriculum. This is a particularly South African phenomenon, because there is no apparent evidence that the OBE system is informed by the relationship between cognitive development, reading and writing. Nor 
is there a practical framework for how learner's literacy development progresses from word reading to complex reading comprehension and academic literacy requirements of grade 4 and above. In most cases, the OBE system is poorly understood (Heugh, 2002, 2010). A poignant example of this is the study done by Reeves et al., (2008: 52) of teacher's perceptions of the OBE system. Teachers claimed that,

The OBE approach means that we do not need to teach reading and writing.

Reeves et al., (2008) also found very little evidence of reading or writing and no evidence was found of learners taking home reading materials in any of the primary schools sampled. Thus, although the communicative language might appear attractive and easier to implement, learners need to be able to read if they are to read with a critical mind. Therefore, an understanding of how children learn and components of teaching (i.e. the actual teaching of reading and writing), needs to be considered, irrespective of a child's L1 or L2 reading situation (Heugh, 2010).

In this study, different combinations of phonological units were predictors of word decoding and reading comprehension. Thus, although word reading forms the basis for developing reading comprehension ability, reading comprehension requires deriving the meaning of particular sentences as well as text-modelling processes that are based on general knowledge and inference drawing ability (Hulme \& Snowling, 2009). Therefore, scaffolded teaching procedures that include, predicting, questiongenerating, summarising, and monitoring the comprehension process, first in the particular text and then more generally (cf. Chamot, 2005 for a review), would be important to enhance English reading comprehension in both groups of learners.

The gap between English word reading and reading comprehension in the EL1 and EL2 groups, however, also merits attention. Leaner's better word reading than reading comprehension ability, suggests that predominate phonological decodingoriented strategies might be sufficient for word decoding, though not fully successful because English is more predictable at the level of groups of letters than individual letter sequences. Further, the fact that the culture of the emergent Zulu-English bilinguals Zulu (L1), was restricted to spoken language, may have had a strong bearing on reading practices in their English (L2) (Cummins, 1979, 1999), and might have led to difficulty in answering comprehension questions for these learners. That is, there is a 'short-circuit' effect for learners whose level of language proficiency is too low to make efficient reading comprehension possible.

For language-policy-in-education, the results provide broad support for Heugh's (2002, 2010) perspective that the choice between English or an African language (including Afrikaans) is a false dichotomy, because developing the L1 and L2 in conjunction is the best way to ensure the successful learning of a second-language. Even if in a dual medium approach were to implemented, language attitudes cannot change overnight, and can only change if the social-linguistic standings of a language spoken in the community changes, whereby the communities must be perceived to have become 'successful' (Webb, 1999). A good example of this is the history of Afrikaans. Whilst the English-speaking Government as well as the Dutch-speaking 
cultural leaders were strongly against the use of Afrikaans in public life, community leaders, such as teachers, and a few church leaders, were the driving force behind promoting the Afrikaans language, and eventually led to the written form of the language being developed, published literature, and over time Afrikaans developed as a language of educational, economic and political power. A similar process is needed in the case of the Zulu language (Webb, 1999). Moreover, a single generalised languagein-education policy is ill-advised. Rather, different language-in-education policies may need to be implemented for different linguistic groups (Webb, 1999).

The moderate-to-large effect size results demonstrated for the statistical differences between the two group of learners for PA and reading measures, differs to findings of previous studies who reported failing to account for differences in the EL2 reading process from that of the EL1 process (e.g. Chiappe \& Siegel, 1999; Low \& Siegel, 2004); is of significance from a theoretical perspective. This can be attributed to two methodological problems in previous studies: the use of a global PA measures in which skill components are confounded and the comparison of bilingual children who differ in their bilingual experience (Bernhardt, 2003). From the results of this study assessment practitioners, need to consider the child's linguistic background and L1 and/or L2 education context as an influence on bilingual learner's L2 reading achievement. For education evaluation, the results suggest that simply taking English language tests in a bilingual context, though recommended by some researchers (e.g. Everatt, Smythe \& Ocampo, 2000) may be of limited value. Even with emergent bilingual children for whom English is the language of reading instruction, testing in the first language may be necessary, or taking into consideration that reading achievement in a second-language unlike a first-language involves more than one language and the interplay between them. Hence, considering interactions between languages, the degree to which levels of proficiency in certain languages and reading achievement and phonological processing are related, as well as how level of bilingualism and the orthographic transparency of the language of reading instruction and language of the home could influence performance on these tasks, should be part of a studies' design, where possible.

PA and reading achievement differences between the English (L1) and English (L2) groups though interesting and valuable from a theoretical perspective, also imply that it is highly unlikely that equity of educational outcome can be achieved. In South Africa, the high prestige of English and the negative social meaning of the Zulu language has resulted in a strong preference for English as medium of instruction. Consequently, schools adopt an English medium language-in-education policy. Such a policy, however, appears to present a serious problem since emergent bilingual, English (L2) is not adequately well-developed for using it as language of learning and teaching. In other words, English acts as an obstacle to educational development, and thus is not offering access to the competitive global job market that is perceived to be associated to English by Zulu parents (Heugh, 2010). Instead, to become and remain proficient in English, emergent bilingual, Zulu-English speaking children need early reading instruction in Zulu and reading instruction in English. For this to happen, parents, and teachers need 
to be made cognisant of the relationship between the child's L1 and L2, and thus need to be made aware of arguments in favour of using Zulu as a medium of instruction alongside English. In this regard, Heugh (2002) points out that there are a number of L1 Zulu speaking teachers who could be deployed to teach the emergent bilingual Zulu-English leaner's at no extra cost to the education system. Hence, a more effective implementation of a multilingual pedagogy requires tailoring a language-in-education policy to capitalise on strengths of learners and teachers and support of L1 alongside L2 instruction. The results of such a development will be a corpus of learners who will be able to cope adequately with academic language skills and will be empowered to read to their full potential.

\section{Conclusion}

Phonological processing skills are thought to contribute to language-learning and literacy development and overall level of academic achievement; these skills serve as a building block upon which all other learning takes place. Differences in phonological processing skills might subsequently result in differences in literacy achievement, which in turn impacts on a country's political, educational and social future. Children with poor literacy skills are at a disadvantage in engaging cognitively and socially in a world that is ever-changing and competitive (Bialystok, 2007; Heugh, 2010). Yet, the different orthographic and phonological systems of different languages may militate against generalising across languages and also exacerbates the problem of bilingual education such as is current in urban South Africa.

\section{References}

Anthony, J. L. \& Lonigan, C. J. (2004). The nature of phonological awareness: Converging evidence from four studies of preschool and early school children. Journal of Educational Psychology, 96: 43-55.

Baker, C. (2006). Foundations of bilingual education and bilingualism, 4th ed. Clevedon: Multilingual Matters.

Benson, C. J. (2002). Real and potential benefits of bilingual programmes in developing countries. International Journal of Bilingual Education and Bilingualism, 5(6): 303-317.

Bernhardt, E. (2003). Challenges to reading research from a multilingual world. Reading Research Quarterly, 38(1): 112-117.

Bialystok, E. (2002). Acquisition of literacy in bilingual children: a framework for research. Language Learning, 52: 159-199.

Bialystok, E. (2007). Language acquisition and bilingualism: consequences for a multilingual society. Applied Psycholinguistics, 28(3): 393-397.

Bialystok, E., Luk, G. \& Kwan, E. (2005). Bilingualism, biliteracy, and learning to read: Interactions among language writing systems. Scientific Studies of Reading, 9(1): 43 -61. 
Bradley, L. \& Bryant, P. (1983). Categorising sounds and learning to read a causal connection. Nature, 301: 419-421.

Buthelezi, Z. (2003). Researchers, beware of your assumptions! The unique case of South African education. Paper presented at the International Reading Association Multilanguage Literacy Symposium Edinburgh, Scotland.

Cardoso-Martins, C. (1995). The reading abilities of beginning readers' of Brazilian Portuguese: implications for a theory of reading acquisition. Scientific Studies of Reading, 5: 289-317.

Chamot A. D. (2005). Language learning strategy instruction: current issues and research. Annual Review of Applied Linguistics, 25: 112-130.

Chiappe, P. \& Siegel, L. S. (1999). Phonological awareness and reading acquisition in English- and Punjabi-speaking Canadian children. Journal of Educational Psychology, 91(1): 20-28.

Cohen, J. (1988). Statistical power analysis for the behavioural sciences, 2nd. ed. Hillsdale: NJ, Erlbaum.

Cope, A. T. (1983). A comprehensive course in the Zulu Language. Durban: University of Natal.

Coolican, H. (2004). Research methods and statistics in psychology, 4th ed. London: Hodder \& Stoughton.

Cummins, J. (1979). Linguistic interdependence and the educational development of bilingual children. Review of Educational Research, 49: 221-251.

Cummins, J. (1999). Alternative paradigms in bilingual education research: does theory have a place? Educational Researcher, 28(7): 26-32.

D’Anguilli, A., Siegel, L. \& Serra, E. (2001). The development of reading in English and Italian bilingual children. Applied Psycholinguistics, 22: 479-507.

Department of Education. (2002). Revised National Curriculum Statements Grades R-9. Government Gazette, 443(23406): 1-20.

Ehri, L. C., Nunes, S. R., Stahl, S. A. \& Willows, D. M. (2001). Systematic phonics instruction helps students learn to read: evidence from the National Reading Panels' meta-analysis. Review of Educational Research, 71: 393-447.

Everatt, J., Smythe, I. \& Ocampo, D. (2000). Dyslexia screening procedures and bilingualism. Dyslexia, 6: 42-56.

Goswami, U. (2000). Phonological representations, reading development and dyslexia: Towards a cross-linguistic framework. Dyslexia, 6: 133-151.

Goswami, U. \& Bryant, P. (1990). Phonological Skills and Learning to Read. New Jersey: Erlbaum. 
Greenop, K. T. (2004). The development of literacy skills in Zulu and Northern Sotho speaking children: a cognitive-neuropsychological investigation. Johannesburg: Unpubl. doctoral thesis, University of the Witwatersrand.

Hays, W. L. (1994). Statistics, 5th ed. Belmont, CA: Wadsworth.

Heugh, K. (2002). The case against bilingual and bilingual education in South Africa: laying bare the myths. Perspectives in Education, 20(1): 171-196.

Heugh, K. (2010). Into the cauldron: interplay of indigenous and globalised notions of literacy and language education in Ethiopia and South African. Language Matters, 40(2): 166-189.

Hulme C. \& Snowling, M. J. (2009). Developmental disorders of language learning and cognition. United Kingdom: John Wiley \& Sons.

Low, P. B. \& Siegel, L. S. (2005). A comparison of the cognitive processes underlying reading comprehension in native English and ESL speakers. Written Language and Literacy, 8(2): 207-223.

Mesthrie, R. (1995). Language and social history: studies in South African sociolinguistics. Cape Town: David Philip.

Neale, M. D. (1997). Neale analysis of reading ability-revised (NARA-II). Windsor, England: NFER.

Naidoo, Y. (2003). A developmental profile of speech sound and syllable acquisition in Zuluspeaking children. Unpubl. master's thesis, University of Pretoria: South Africa.

Poulos, G. \& Msimang, C. T. (1998). A linguistic analysis of Zulu. Johannesburg: Horizon.

Raven, J., Raven, J. C. \& Court, J. H. (1998). Coloured progressive matrices. Oxford: Oxford Psychologist Press.

Reeves, C. R., Heugh, C. H. Prinsloo, C., MacDonald, T., Netshitangani, H., Alldou, D., Diedericks, G. \& Herbst. (2008). Evaluation of literacy in the primary schools of the Limpopo province. Pretoria: Human Sciences Research Council in association with the University of Limpopo.

Rosner, J. (1979). Helping children overcome learning difficulties. New York: Walker and Company.

Smith, M. W., Dickinson, D. K., Sangeorge, A. \& Anastasopoulos, L. (2002). Early language and literacy classroom observation. Brookes: Baltimore.

Spencer, L. H. \& Hanley. J. R. (2003). Effects of orthographic transparency on reading and phoneme awareness in children learning to read in Wales. British Journal of Psychology, 94: 1-28.

Snow, C. E., Burns, M. S. \& Griffith, P. (eds.) (1998). Preventing reading difficulties in young children. Washington, DC. National Academic Press. 
Suzman, S. (1996). Acquisition of noun classes in related Bantu languages. In C. E. Johnston \& J. H. V. Gilnbert (eds.), Children's language, 9: 87-104. New Jersey: Lawrence Erlbaum Associates.

Thorstad, G. (1991). The effect of orthography on the acquisition of literacy skills. British Journal of Psychology, 82: 527-537.

Treiman, R. \& Zukowski, A. (1996). Children's sensitivity to syllables, onsets, rimes and phonemes. Journal of Experimental Child Psychology, 61: 193-215.

Verhoeven, L. (2007). Early bilingualism, language transfer and phonological awareness. Applied Psycholinguistics, 28: 425-439.

Wimmer, H. (1993). Characteristics of developmental dyslexia in a regular writing system. Applied Psycholinguistics, 14: 1-33.

Wimmer, H. \& Goswami, U. (1994). The influence of orthographic consistency on reading development: Word recognition in English and German children. Cognition, 51: 368-394.

Webb, V. (1999). Multilingualism in democratic South Africa: the over-estimation of language policy. International Journal of Educational Development, 19: 351-366.

Weschler, D. (2005). WIAT-II Technical and Interpretive Manual. San Antonio, TX: Harcourt Assessment, Inc. 